\title{
A recurrence of advanced malignant sex cord tumor with annular tubules: case report
}

\author{
Ping Zheng ${ }^{1,2}$, Jinhua Leng ${ }^{1}$, Jinghe Lang ${ }^{1}$ \\ ${ }^{1}$ Department of Obstetrics and Gynecology, Peking Union Medical Hospital, Peking Union Medical College Hospital, Chinese Academy of Medical \\ Sciences, Beijing 100730, China; ${ }^{2}$ Department of Gynecology, Liuzhou Maternity and Child Healthcare Hospital, Guangxi University of Science and \\ Technology, Liuzhou 545000, China \\ Correspondence to: Jinghe Lang, MD. Department of Obstetrics and Gynecology, Peking Union Medical Hospital, Peking Union Medical College \\ Hospital, Chinese Academy of Medical Sciences, Beijing 100730, China. Email: langjh@hotmail.com.
}

\begin{abstract}
Sex cord tumor with annular tubules (SCTAT) is a rare special type of ovarian sex cordstromal tumor, which has not achieved a standard treatment regimen currently. The aim of this report was to provide possible reference for treatment and supervision of recurrent advanced malignant SCTAT. A 33-year-old unmarried female with malignant SCTAT emerged recurrence after the cytoreductive surgery but preserved fertility and postoperative chemotherapy (3 cycles PEB regimens) 12 months. She was performed preoperative adjuvant chemotherapy (3 cycles, paclitaxel liposome and nedaplatin) before the third operation of secondary cytoreductive surgery, followed by platinum-based chemotherapy (7 cycles, TC regimens) timely because of dissatisfactory efficacy under the evaluation in combination with prior comprehensive treatment and PET/CT. The surgical stage was IIIB. She acquired complete remission after therapy and needed to regularly follow up every 3-6 months for 2 years and every 6-12 months after 2 years. There was no evidence of malignant SCTAT recurrence at the follow-up 24 months past the third surgery. No abnormality was found in other examination indexes, and menstruation was regular. The regular supervision of the patient was continually performed. The diagnosis of recurrent advanced malignant SCTAT was established on history and final pathology examination, whose prognosis mainly depended on the appropriate strategy of surgery and chemotherapy regimens. The classical TC regimens could be considered if the efficacy of other chemotherapy regimens were unsatisfactory. Regular follow-up was essential.
\end{abstract}

Keywords: Sex cord tumor with annular tubules (SCTAT); recurrence; malignant; therapy

Submitted Oct 05, 2019. Accepted for publication Dec 05, 2019.

doi: $10.21037 /$ tcr.2019.12.56

View this article at: http://dx.doi.org/10.21037/tcr.2019.12.56

\section{Introduction}

The morbidity of sex cord-stromal tumor may be only approximately $1.2-2 \%$ of primary ovarian cancer (1). However, sex cord tumor with annular tubules (SCTAT) is a rare special type of ovarian sex cord-stromal tumor, and accounts for approximately $6 \%(2,3)$, which histomorphology is considered as between a supportive cell tumor and a granulosa cell tumor. SCTAT is reported that about one third of patients experience Peutz-Jeghers syndrome (PJS) with mucocutaneous pigmentation in special locations and gastrointestinal tract polyposis, whose form is usually benign and has excellent prognosis through surgery. However, SCTAT without PJS may have a 15-20\% malignancy rate and malignant behavior with metastases might be examined in approximately $20 \%$ of patients (4), which is a most common phenomenon in women of reproductive age. The tumors both may express the hyperestrogenic manifestations, regardless of the clinical form.

Although SCTAT is a rare disease, it's not scary. The patient with SCTAT related to non-PJS is considered to emerge malignant behavior with recurrence and metastases, which also still has a good prognosis via excellent surgery 
and chemotherapy $(5,6)$. However, currently there is no standard treatment in advanced malignant SCTAT. Hence, we report a surgical stage IIIB recurrent case with SCTAT related to non-PJS, to provide possible reference for therapy and supervision. We present the following case in accordance with the CARE-Guideline (7).

\section{Case presentation}

An unmarried female, 33-year-old, gravida-1-para-0, without a history of ovarian and uterine tumors, emerged menstrual disorder for 6 months, presenting with irregular menstrual cycles (cycle is $15-40$ days, used to be 30 days) and heavy menstrual bleeding, without abdominal pain and mucocutaneous pigmentation visually. The patient was discovered a cyst sized approximately $4 \mathrm{~cm} \times 5 \mathrm{~cm}$ in right ovary by transvaginal ultrasonography. Finally, she received a laparoscopic ovarian cystectomy on account of the ovarian cyst achieved the surgical requirements. Postoperative pathology reported ovarian granulosa cell tumor. There was no adjuvant therapy such as postoperative chemotherapy. During the follow-up 8 months after the first surgery, the right ovary was recurred a $5 \mathrm{~cm} \times 6 \mathrm{~cm}$ mass by pelvic ultrasonography, which suggested cystic solid and rich blood flow sign, but without the hyperestrogenic manifestations. This recurrence of cyst was considered as the malignant tumor. Hence, the second surgery was made to perform with laparoscopic right salpingo-oophorectomy and greater omentectomy and bilateral pelvic and paraaortic lymphadenectomy and left appendix and suspicious lymph nodes resection. Intraoperatively, a mass with sized about $5 \mathrm{~cm} \times 5 \mathrm{~cm}$ was seen in the right ovary, several vesicles were seen outside the right fallopian tube, multiple small lesions were found on the intestinal tube surface, but the anatomy of the left fallopian tube and ovary was normal. The lesions were removed completely. Postoperative pathology evaluation of the tumor demonstrated the neoplasm was ovary SCTAT, additionally, 27 lymph nodes from pelvic and partial omentum were not metastasized by the tumor. The surgical stage was IIIB after operation, then $100 \mathrm{mg}$ of nedaplatin intraperitoneal chemotherapy was performed. Subsequently adjuvant regimens including 3 cycles PEB (cisplatin, etoposide and bleomycin) intravenous chemotherapy was received. The treatment process was successful and the side effects were controllable. Thereafter, she followed up regularly every 3 months. The above treatment was conducted in the local hospital of this patient.

One year after the second operation, she returned to the hospital for examination because the emergence a symptom of irregular vaginal bleeding. Serological examinations showed the serum levels of estradiol reached $164 \mathrm{pg} / \mathrm{mL}$, but levels of other hormones including progestin, androgen, luteinizing hormone (LH), follicle stimulating hormone $(\mathrm{FSH})$, prolactin were in the normal range. Then, the multiple little lesions on the surface of pelvic and abdominal viscera were recurred by MRI examination, which were considered clinical relapse. Hence, the pre-chemotherapy including 3 cycles paclitaxel liposome and nedaplatin was decided by the professors from department of gynecological oncology in Peking Union Medical College Hospital (PUMCH). After the pre-chemotherapy, she was recommended to detect PET-CT examination, in order to further measure the disease and followed therapy. The result exhibited that the right posterior lobe of the liver was occupied, and the metabolism was increased, and left pelvic soft tissue lesion, increased metabolism, which strongly supported metastasizing lesions. The third operation, secondary cytoreductive surgery, was planned through the evaluation in combination with prior comprehensive treatment and PET-CT. This time the aim of this surgery was to remove the visible tumors of the pelvic and abdominal cavity completely. Postsurgical pathology reported that these lesions, including left abdominal wall, upper uterine wall, posterior uterine wall, left broad ligament, colonic surface, subhepatic retroperitoneal, retroperitoneal space, retroperitoneal surface, rectal surface, mesenterium surface, anterior abdominal wall, were consistent with the diagnosis of malignant SCTAT (see the Figure 1). She received 7 cycles postoperative chemotherapy of TC (paclitaxel and carboplatin) regimens. The patient acquired complete remission after therapy during followup stage. The whole treatment process flow was shown in Figure 2.

The patient would be re-examined in Gynecological Oncology Outpatient Department after end chemotherapy 1 month. The serum levels of estradiol had decreased to the normal range value. The abdominal and pelvic Ultrasound examination also found no abnormalities. Subsequent supervision should be followed every 3-6 months for 2 years and every 6-12 months after 2 years. Now the patient has been followed up 2 years, who would be monitored longterm. The monitoring involved physical exam including pelvic exam, serum tumor makers (e.g., estrogen), and radiographic imaging (e.g., chest $\mathrm{X}$-ray) or abdominal and pelvic ultrasound for evaluating the mass recurrence. The examinations of chest/abdominal/pelvic CT, MRI, PET/CT 

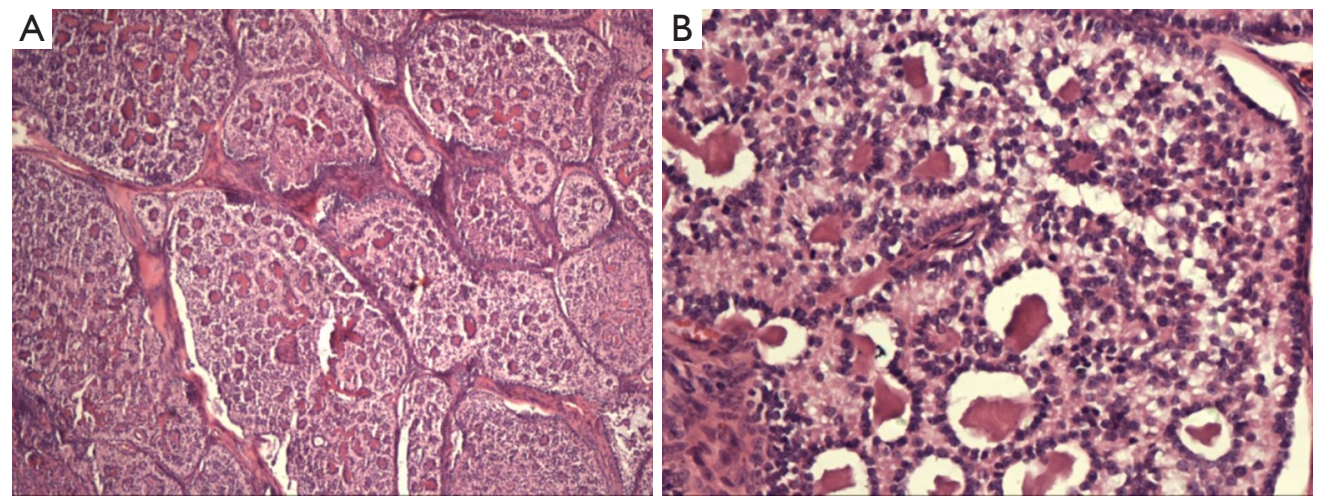

Figure 1 Sex cord tumor with annular tubules (A: HE, $\times 40$; B: HE, $\times 200$ ).

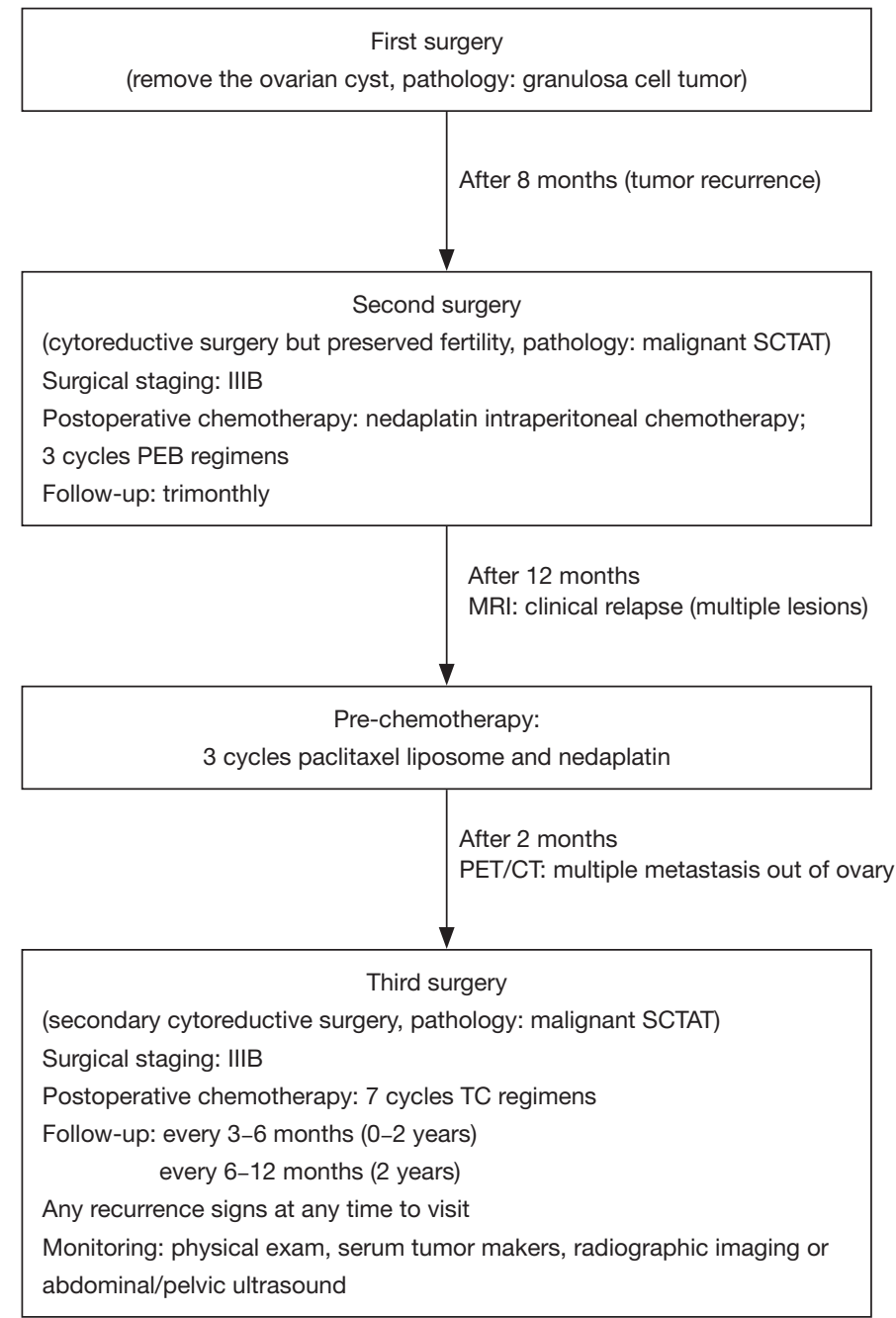

Figure 2 The therapeutic process flow. SCTAT, sex cord tumor with annular tubules. 
or PET would be performed when a suspicious abnormality was found in the previous basic inspection.

Over the follow-up 24 months past the third surgery, there was no evidence of malignant SCTAT recurrence. No abnormality was found in other test indexes, and menstruation was regular.

\section{Discussion}

Clinical presentations of sex cord tumors with annular tubules include irregular vaginal bleeding or menstrual disorder, hyperestrinism symptoms, and the presence of an abdominal or pelvic mass $(5,8,9)$. Childbearing female was a vulnerable population to suffer SCTAT, however the young girl was reported only in sporadic cases $(10,11)$, usually presenting malignant behaviors and causing precocious puberty because of secreting estrogenprogesterone $(10,12,13)$. The form with PJS or without PJS was identified as two characteristic manifestations. SCTAT with PJS was usually benign and had a genetic predisposition. Surgical resection of the lesion or could result in good remission and a good prognosis. SCTAT without PJS had a malignant tendency, approximately $22 \%$, which seemed to spread mainly through the lymphatics with typical locations of tumor metastasis being the pelvic tissues, particularly in high-order lymph nodes, such as para-aortic and supraclavicular lymph nodes (14). The peritoneum, omentum majus, kidney, liver, lung and other organs might be prone to metastasis and recurrence. In spite of metastasis, the fertility could be retained (15). Though the serum tumor markers such as inhibin, CD99, MIS, estrogen receptor, progesterone receptor have been studied as diagnostic moleculars and supervision of clinical prognosis of the tumor post-operation (16), these laboratory examinations were still nonspecific. This disease should be differentiated from granulosa cell tumor and other endocrine tumors of ovary. Pelvic ultrasound examination could be used as an auxiliary detection tool. This case first diagnosis was classified as ovarian granulosa cell tumor in her local hospital because of similar secretory hormone characteristics to SCTAT and pelvic mass. Hence the final diagnosis still depended on pathology characterized by simple or complex annular tubes (17) (see Figure1A,B), even providing a disease history might be necessary.

Surgical staging and treatment were similar to other malignant sex cord-stromal tumors and epithelial ovarian cancer. Patients with stage II to IV tumor were recommended cisplatin, etoposide and bleomycin as postoperative chemotherapy, which resulted in pathological complete remission (18). Recurrence treatment included docetaxel, paclitaxel/ifosamide, and paclitaxel/carboplatin. Hormone recurrence therapy included aromatase inhibitors, leuprolide, and tamoxifen. Secondary cytoreductive surgery might also be considered (19). In our case, this patient appeared multiple recurrent and metastatic lesions in the abdominal and pelvic cavity via MRI examination no more than 12 months after the second operation and PEB regimens. Hence, switch to paclitaxel liposome and nedaplatin (3 cycles) as the adjuvant chemotherapy. However, the multiple lesions were not decreased by PET/ CT evaluation, considering the unsatisfactory effect of the chemotherapy regimens. Therefore, the secondary cytoreductive surgery was decided through negotiations by the gynecologic oncologists. Subsequently, postoperative adjuvant chemotherapy was switched to the classical TC regimens, without hormone therapy. Ultimately, our patient acquired complete remission and disease-free status after 7 cycles of chemotherapy, indicating that the current therapy was suitable for this patient. Therefore, we believed that the classical TC regimens could be considered if the efficacy of other chemotherapy regimens were unsatisfactory in recurrent advanced malignant SCTAT.

Malignant SCTAT has a long-term risk of recurrence. Kulkarni (20) has reported the time of first recurrence in patients with malignant SCTAT ranges from 3 months to 20 years. Shen et al. (3) also reported the relapse and metastasis rate at first surgery was $20 \%$. In our case, she appeared multiple metastasis lesions, which located in the abdominal wall, upper uterine wall, posterior uterine wall, left broad ligament, colonic surface, subhepatic retroperitoneum, retroperitoneal space, retroperitoneal surface, rectal surface, mesorectal mesentery, and anterior peritoneum after comprehensive staging surgery 12 months during the regular supervision. Unfortunately, these lesions were confirmed as metastatic malignant SCTAT by postoperative pathology. Fortunately, the clinical relapse was detected early and timely surgical treatment and appropriate chemotherapy regimens were performed to allow the disease to achieve complete remission.

The mortality of SCTAT is unclear. The prognosis of SCTAT needs to combine with the tumor clinical stage and the nature of neoplasm with benign or malignant. The prognosis of recurrent malignant SCTAT is decided based on the metastatic sites, surgical stage and treatment strategy (21). Qian et al. (5) demonstrated a 100\% survival rate in 5 year, while their median progression-free survival 
time was 97.8 months by a retrospective study, which showed the overall good prognosis. The annual surveillance for 8-10 years with or without ultrasound and tumor makers serology (estradiol and/or progestin) depending on clinical suspicion was recommended $(4,22)$. In our case, regular supervision has persisted in 2 years. In duration, the physical exam, hormone examination and abdominal/pelvic ultrasonography have been performed every 3-6 months from the last discharge. The disease-free status included no abdominal pain or disorder vaginal bleeding, and no other recurrence signs were proven by monitoring. This patient has been followed up regularly for long-term.

\section{Acknowledgments}

Funding: We thank the financial support from the Chinese Academy of Medical Sciences Initiative for Innovative Medicine (CAMS-2017-I2M-1-002).

\section{Footnote}

Conflicts of Interest: All authors have completed the ICMJE uniform disclosure form (available at http://dx.doi. org/10.21037/tcr.2019.12.56). The authors have no conflicts of interest to declare.

Ethical Statement: The authors are accountable for all aspects of the work in ensuring that questions related to the accuracy or integrity of any part of the work are appropriately investigated and resolved. All procedures performed in studies involving human participants were in accordance with the Declaration of Helsinki (as revised in 2013). Written informed consent was obtained from the patient for publication of this manuscript and any accompanying images.

Open Access Statement: This is an Open Access article distributed in accordance with the Creative Commons Attribution-NonCommercial-NoDerivs 4.0 International License (CC BY-NC-ND 4.0), which permits the noncommercial replication and distribution of the article with the strict proviso that no changes or edits are made and the original work is properly cited (including links to both the formal publication through the relevant DOI and the license). See: https://creativecommons.org/licenses/by-nc-nd/4.0/.

\section{References}

1. Quirk JT, Natarajan N. Ovarian cancer incidence in the
United States, 1992-1999. Gynecol Oncol 2005;97:519-23.

2. Bercaw JL, Sanchez J, Byrd RH, et al. Sex cord tumor with annular tubules in a young adolescent with Von Willebrand's disease. J Pediatr Adolesc Gynecol 2010;23:e111-4.

3. Shen K, Wu PC, Lang JH, et al. Ovarian sex cord tumor with annular tubules: a report of six cases. Gynecol Oncol 1993;48:180-4.

4. Jaegle WT, Keyser EA, Messersmith L, et al. Extraovarian sex cord tumor with annular tubules discovered arising from a leiomyoma. Gynecol Oncol Rep 2018;26:17-20.

5. Qian Q, You Y, Yang J, et al. Management and prognosis of patients with ovarian sex cord tumor with annular tubules: a retrospective study. BMC Cancer 2015;15:270.

6. Gibbon DG. Conservative management of sex cord tumors with annular tubules of the ovary in women with Peutz-Jeghers syndrome. J Pediatr Hematol Oncol 2005;27:630-2.

7. Riley DS, Barber MS, Kienle GS, et al. CARE guidelines for case reports: explanation and elaboration document. J Clin Epidemiol 2017;89:218-35.

8. Nosov V, Park S, Rao J, et al. Non-Peutz-Jeghers syndrome associated ovarian sex cord tumor with annular tubules: a case report. Fertil Steril 2009;92:1497.e5-8.

9. Meserve EE, Nucci MR. Peutz-Jeghers Syndrome: Pathobiology, Pathologic Manifestations, and Suggestions for Recommending Genetic Testing in Pathology Reports. Surg Pathol Clin 2016;9:243-68.

10. Ghribi A, Ben Slama A, Gasmi M, et al. Ovarian sex cord tumor with annular tubules in children. Tunis Med 2014;92:762-3.

11. Sánchez Zahonero J, Ruiz Dominguez JA, Sanchez Zahonero $\mathrm{S}$, et al. A very rare cause of peripheral precocious puberty in a girl: ovarian sex cord tumor with annular tubules. Arch Argent Pediatr 2017;115:e179-82.

12. Zung A, Shoham Z, Open M, et al. Sertoli cell tumor causing precocious puberty in a girl with Peutz-Jeghers syndrome. Gynecol Oncol 1998;70:421-4.

13. Ruibal Francisco JL, Palomino Bueno MC, Rivilla Parra F, et al. [Sexual precocity in a 7-year old girl, due to a tumor of the ovarian sex cord with annular tubules (SCTAT of Scully)]. An Esp Pediatr 1998;48:409-11.

14. Momin YA, Kulkarni MP, Pandav AB, et al. Non PeutzJegher syndrome associated malignant sex cord stromal tumor with annular tubules. Int J Appl Basic Med Res 2013;3:126-8.

15. Barker D, Sharma R, McIndoe A, et al. An unusual case of sex cord tumor with annular tubules with malignant 
transformation in a patient with Peutz-Jeghers syndrome. Int J Gynecol Pathol 2010;29:27-32.

16. Roth LM. Recent advances in the pathology and classification of ovarian sex cord-stromal tumors. Int J Gynecol Pathol 2006;25:199-215.

17. Young RH. Ovarian sex cord-stromal tumours and their mimics. Pathology 2018;50:5-15.

18. Puls LE, Hamous J, Morrow MS, et al. Recurrent ovarian sex cord tumor with annular tubules: tumor marker and chemotherapy experience. Gynecol Oncol 1994;54:396-401.

19. Korach J, Perri T, Beiner M, et al. Promising effect of aromatase inhibitors on recurrent granulosa cell tumors. Int J Gynecol Cancer 2009;19:830-3.

Cite this article as: Zheng P, Leng J, Lang J. A recurrence of advanced malignant sex cord tumor with annular tubules: case report. Transl Cancer Res 2020;9(3):2089-2094. doi: 10.21037/ tcr.2019.12.56
20. Kulkarni N. Recurrence of Non-Syndromic Sex Cord Stromal Tumor with Annular Tubules of Ovary: Case Report. Iran J Pathol 2015;10:61-4.

21. Ravishankar S, Mangray S, Kurkchubasche A, et al. Unusual Sertoli Cell Tumor Associated With Sex Cord Tumor With Annular Tubules in Peutz-Jeghers Syndrome: Report of a Case and Review of the Literature on Ovarian Tumors in Peutz-Jeghers Syndrome. Int J Surg Pathol 2016;24:269-73.

22. Komiyama S, Katabuchi H, Mikami M, et al. Japan Society of Gynecologic Oncology guidelines 2015 for the treatment of ovarian cancer including primary peritoneal cancer and fallopian tube cancer. Int J Clin Oncol 2016;21:435-46. 